\title{
Classical Grains and Platt Particles: Structural Characteristics and Interaction With Galactic Radiation
}

\author{
J. A. MCMilLaN \\ Argonne National Laboratory \\ Argonne, Illinois
}

DURING THE LAST . 20 YeARS much work has been done, both theoretical and experimental, that has improved the understanding of the nature and characteristics of interstellar grains; most of it, however, has not been primarily involved in this particular field. Unfortunately, the problem is extremelv complex. As recentlv pointed out (ref. 1), an analysis of the nature and growth of interstellar grains calls for consideration of: (1) nucleation of condensing vapors; (2) mechanisms of crystal growth; (3) chemical reactions at low temperature including surface reactions of free radicals; and (4) radiation chemistry of solids. Simultaneous analysis of all these factors seems to be, at the present time, a hopeless endeavor. With the exception of radiation effects, separate analyses of each one have extensively been made. So far, however, it seems that combined effects cannot be overlooked. Partial aspects of the problem are discussed in this paper. The treatment of radiation is essentially exploratory, and its relative success ought not to blind us to the uncertainty of the data and to the speculative character of some of the assumptions.

\section{CHEMICAL PROCESSES}

Chemical reactions in interstellar grains must be intimately related to the mechanisms of growth. In the simplified assumption that growth takes place by incorporating with the grain all hitting atoms heavier than hydrogen (with the exception of the rare gases) plus the hydrogen atoms needed to saturate the lowest weight molecules, it is necessary to account for two factors; namely: (1) the evolution of heat accompanying chemical reactions; and (2) the possible existence of activation bar- 
riers. The evolution of heat would warm up a local region favoring partial vaporization and structural rearrangement. The existence of activation barriers would deny the initial statement that all encounters must lead to chemical combination.

According to the simple picture given in the previous paragraph, chemical reactions would essentially be addition reactions between radicals, i.e., $\mathrm{OH}+\mathrm{H} \rightarrow \mathrm{H}_{2} \mathrm{O}, \mathrm{CH}+\mathrm{H} \rightarrow \mathrm{CH}_{2}, \mathrm{~N}+\mathrm{H} \rightarrow \mathrm{NH}$, and so on. Eventual recombination of hydrogen atoms, adsorbed and diffusing on the grain surface, should yield molecular hydrogen that would soon evaporate, releasing to the grain a fraction of the recombination energy. (See ref. 2.) All these reactions should proceed, in the gaseous state, with little or no activation energy at all. Radicals attached to the grain, however, should behave differently, and activation energies of as much as $1 \mathrm{kcal} / \mathrm{mole}$ could reasonably be expected. Under such circumstances, the rate of growth could be reduced by a large factor. Subtraction reactions of the type $\mathrm{CH}_{4}+\mathrm{H} \rightarrow \mathrm{CH}_{3}+\mathrm{H}_{2}$, that could lead to polymerization and further cross-linking, would be much less probable since they should have larger activation energies. Mechanisms of polymerization and cross-linking could occur, however, as a consequence of reactions among radicals other than hydrogen. Since most heavy atoms, with the exception of the rare gases, have rather low ionization potentials, increasing consideration has lately been given to reactions involving ionic species. Whether or not their larger cross section for chemical reactions could compete with the overwhelming abundance of hydrogen has not yet been proved. In one way or another, it is clear that the simplified theory of growth is unsubstantial, and that more sophisticated mechanisms must be sought to account for the growth and nature of interstellar grains.

Radicals trapped in a classical grain seem to be improbable. Hydrogen atoms would not only attach to the grain but also diffuse through it with ease, pairing unpaired electrons with very small activation energy. A Platt particle, on the other hand, could indeed have unfilled electron bands since the hydrogen atoms would not enter the big, unsaturated molecule but probably react at the surface, if at all. With the recent discovery of the chemistry of rare gases, one is tempted to introduce them in the pattern of growth. Their potential role is discussed in reference 3. Such reactions would seem to be very improbable with the light elements helium and neon, whose abundance is significant, although their bearing in ionic reactions could be important. (See ref. 3.) Reactions with the heavier ones, whose abundance is small, are probably unimportant. The field, however, is open. It should be noted that consideration of the interaction of the grain with galactic radiation is imperative. 


\section{STRUCTURAL CHARACTERISTICS OF CLASSICAL GRAINS}

An important question about classical grains is the extent to which their molecules are arranged in a regular pattern resembling the longrange order of crystalline material. Recent experiments presented in references 4 and 5 have provided values for the activation barriers of structural transformations that might occur in a classical grain. If one considers a grain of ice, in order to simplify the treatment one may ask, in view of its low temperature, whether it would be amorphous or crystalline. Low-temperature, low-pressure condensation of water molecules leads to an amorphous state of aggregation that undergoes the following experiences during warmup: (1) a glass transformation at about $140^{\circ} \mathrm{K}$; (2) crystallization in the space group $\bar{F} \overline{4} 3 \mathrm{~m}$ at about $150^{\circ} \widehat{\mathrm{K}}$; and (3) recrystallization in the space group $\mathrm{P6}_{3} / \mathrm{mmc}$ at about $185^{\circ} \mathrm{K}$. Table $\mathrm{I}$, taken from reference 4 , displays the values of the activation barriers of each transformation.

The fundamental difference between these experiments and the process of accretion in a classical grain of ice is that, whereas in the laboratory water molecules are collected and thus the energy corresponding to formation of van der Waals and hydrogen bonds and eventually the excess thermal energy is released, in space this process must be accompanied by the release of heat due to chemical reactions. If one avoids the primordial question of how the first atoms stuck together, one may start with a small grain (nucleus) that will grow by accretion. Formation of chemical bonds upon attachment of an additional atom ( $\mathrm{H}$ or $\mathrm{O}$ ) would warm up the grain above the crystallization temperature unless it were large enough to absorb the energy released by chemical bond formation without a significant temperature rise. In this latter case, it would be necessary to study the structural implications of local warmup.

In order to estimate the limiting size above which only local warmup

TABLE I.-Activation Thermodynamic Functions for Different LowTemperature, Structural Transformations of Water Substance

[From ref. 4]

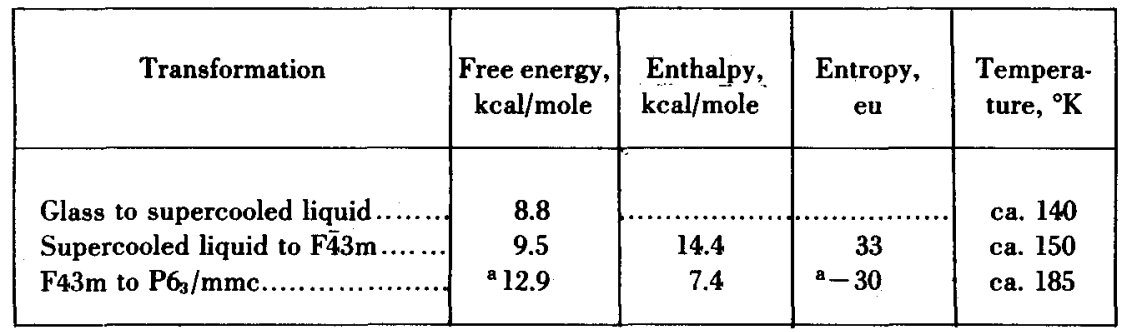

${ }^{\text {a }}$ Fictitious values that probably include a small coefficient of transmission for activation, indicating dislocation-controlled growth. 
could play a significant role, synthesis of a water molecule at the grain surface is assumed to occur with an energy release of 9 electron volts. The problem is further simplified by assuming that the saturated grain first fixes an oxygen atom, and then two hydrogen atoms (that might have been diffusing on the surface), each one releasing about $4.5 \mathrm{eV}$. If it is assumed that the initial temperature of the grain is $20^{\circ} \mathrm{K}$ and that if its temperature is raised by $100^{\circ} \mathrm{K}$ crystallization will occur at a measurable rate, the critical size comes close to 200 water molecules. Such a core should be crystalline and most likely hexagonal since the rise in temperature must have been larger while the grain was smaller.

It should be noted that the energy released by crystallization in the space group $\mathbf{F} \overline{4} 3 \mathrm{~m}$ increases the temperature to a value very close to that at which recrystallization occurs in the space group $\mathrm{P} 6_{3} / \mathrm{mmc}$. Further deposition should be considered for local heating and the seeding effect of the grain. In this case it seems that one has to deal with a competitive process between the half-life of the activated complexes leading to long-range order and the dissipation of heat into the bulk of the grain. It is not known whether dislocations would play a significant role in this type of mechanism since local heating could destroy local order in a region where the chemical attachment takes place. Since the energy released by a single chemical event is enough to warm up a number of molecules able to nucleate collectively, if this effect occurs faster than the dissipation of thermal energy to the bulk of the grain, one imagines the hypothetical grain as hexagonal and polycrystalline.

An alternative that would give utmost importance to the existence of dislocations would be to assume that the surface of the grain is saturated with diffusing hydrogen atoms, and that oxygen atoms are preferentially trapped at or near a dislocation. Chemical combination would take place mainly at the dislocation. If the heat only produced a transitory, reversible process of disorder in the surroundings of the dislocation, one should expect preferential growth of whiskers and platelets. Water molecules formed far from the dislocation could still diffuse toward it and incorporate in the lattice. In the case of crystallization of hydrazine monohydrate, the activation energy for self-diffusion drops has been observed to two-thirds in the molecular layers close to the growing crystal. This result supports the idea of having much smaller activation energies for surface diffusion in a vacuum.

These conclusions cannot be extrapolated without reserve to a classical grain of complex composition, but they nevertheless support the idea of a classical grain with the longest range order compatible with its chemical composition. As in the case of chemical reactions, introduction of galactic radiation completely changes the general pattern since the energies involved in this type of interaction are of the order of $30 \mathrm{eV}$. It is once more obvious that separate treatment of the different factors 
affecting the growth and nature of interstellar grains may be hopelessly misleading.

\section{INTERACTION WITH GALACTIC RADIATION}

Although numerous speculations have been made regarding mechanisms of growth and size-determining factors, not until recently has degradation of grains by galactic radiation been quantitatively considered. (See ref. 6.) A tentative survival equation is derived by taking into account (1) the rate of growth; (2) the radiation dose rate; (3) the specific dose needed for destruction; and (4) the energy released by a single excitation process. No consideration is given to stopping mechanisms other than inelastic collisions.

In the simple theory of growth, the mass $m(t)$ of a grain at age $t$ is

$$
m(t)=k t^{3}
$$

where $k$ is the tridimensional growth-rate constant. The constant $k$ is related to the rate of growth $k_{0}$ (mass per unit area per unit time) by $k \cong 4 k_{0}^{3}$. The surface of the grain has been considered proportional to $m^{2 / 3}$. An elongated, tridimensional grain accounting for polarization phenomena would still follow the rate law expressed by equation (1) with an uncertainty factor of order unity affecting the value of $k$.

The total dose of irradiation at age $t$ is

$$
W=\int_{0}^{t} D^{\prime} m(t) \mathrm{d} t
$$

where $D^{\prime}$ is the dose rate per unit mass. In order to simplify the treatment, the following three assumptions are made:

(1) The parameters $k$ and $D^{\prime}$ have remained constant through the ages.

(2) Cosmic particles lose energy to the grain in discrete bursts of average value $\mathscr{W}_{m}$. In dealing with a single grain, setting equation (2) equal to $W_{m}$ permits evaluation of the probable age at which the single event of interaction will take place.

(3) Complete vaporization of a grain occurs upon single absorption of a specific dose $D_{0}$.

The condition of survival may then be expressed by the inequality

$$
W_{m}<D_{0} m(t)
$$

The probable age at which such an absorption will take place is given by

$$
W_{m}=\int_{0}^{t} D^{\prime} m(t) \mathrm{d} t
$$


There will be, in general, a distribution around the root $t$ of equation (4) accounting for those grains that will absorb $W_{m}$ at earlier and later ages, as well as a distribution of values around $W_{m}$. Introduction of equation (1) into equation (4) and integration results in

$$
W_{m}=\frac{1}{4} k D^{\prime} t^{4}
$$

in view of the first assumption. Elimination of $t$ by an appropriate combination of equations (1), (3), and (5) finally yields the equation of survival

$$
64 k D_{0}^{4} /\left(W_{m} D^{\prime 3}\right)>1
$$

Survival is therefore favored by large values of $k$ and $D_{0}$, as well as by small values of $W_{m}$ and $D^{\prime}$. If the value of $W_{m}$ is not essentially affected in dealing with very small grains - and this may be a weak point of this argument - one could expect a critical size below which interaction with radiation would lead to destruction. If this value is reached before absorption of the burst of energy $W_{m}$, the grain will have a significant chance of surviving; otherwise, it will vaporize. The next step is then to evaluate this size.

Since survival is favored by large values of $k$, it is assumed that growth takes place by incorporating with the grain all hitting atoms heavier than hydrogen (except the rare gases) and the hydrogen atoms needed to saturate the lowest weight molecules. Such an oversimplified theory of growth provides an absolute maximum for the growth-rate constant in $\mathrm{H} I$ regions of average density:

$$
k=6 \times 10^{-23} \mathrm{~g} / \text { megayear }^{3}
$$

by adopting a simplified spherical grain and accepted values for the abundance of the elements and the temperature of the gas in $\mathrm{H} \mathrm{I}$ regions, i.e., $k_{0} \simeq 2.5 \times 10^{-8} \mathrm{~g} / \mathrm{cm}^{2}-\mathrm{Myr}$. (See ref. 3.) The shape of the grain has no significant bearing on the treatment that follows, provided that it grows tridimensionally. A separate treatment is needed for platelets and whiskers in view of their exponential rate of growth.

The specific energy required for complete vaporization is evaluated in reference 7 as

$$
D_{0}=6 \times 10^{21} \mathrm{eV} / \mathrm{g}
$$

A lower value is proposed in reference 6:

$$
D_{0}=10^{21} \mathrm{eV} / \mathrm{g}
$$

with an uncertainty factor of order unity. Since the highest value of $D_{0}$ 
imposes the least stringent condition for survival, the result of reference 7 will be used.

Estimates of $W_{m}$ vary from $30 \mathrm{eV}$ (ref. 6) to as much as $100 \mathrm{eV}$ (ref. 8). This energy is transferred as excitation of a small cluster of molecules and rapidly decays to either thermal motion or chemical dissociation, or both. For a metal, this energy is stored in the form of interstitials and vacancies. (See ref. 9.) The weak point of this treatment is that electronic excitation of a very small grain may result in ionization of the grain by loosing an electron that would carry out a large fraction of $W_{m}$. In the treatment that follows, this possibility is disregarded.

An estimate tending to favor survival is, therefore,

$$
W_{m}=30 \mathrm{eV}
$$

By replacing the figures for the parameters in equation (6), one obtains the value of the maximum specific dose rate compatible with survival:

$$
D_{M}^{\prime}=10^{21} \mathrm{eV} / \mathrm{g} \text {-megayear }
$$

Since the present estimate of the specific dose rate is 0.6 to $2.2 \mathrm{millirad} / \mathrm{hr}$ (ref. 10), i.e.,

$$
D^{\prime}=3.3 \times 10^{20} \text { to } 1.2 \times 10^{21} \mathrm{eV} / \mathrm{g} \cdot \mathrm{Myr}
$$

survival is improbable since the actual value of the growth-rate constant $k$ is likely to be smaller, by orders of magnitude, than the maximum value derived from the simple theory of growth. The $H I$ regions of high density, however, could eventually favor survival because of the weight of $k_{0}$ in the value of $k$, where $k=4 k_{0}^{3}$. The value of $D_{0}$ could not be seriously in error. Moreover, as has been pointed out in reference 6 , it might be smaller, as indicated in equation (9), since destruction of a grain could be accomplished by its splitting into atoms, molecules, and smaller grains. Subject to the restrictions imposed by the indetermination of $W_{m}$ in the case of very small grains, such an argument would strongly favor destruction in view of the weight of the term $D_{0}^{4}$ in the survival equation.

The maximum mass of a classical grain must then be given by

$$
W_{m} / D_{0}=m(t)
$$

Replacing the values for the constants, one arrives at

$$
m(t)=5 \times 10^{-21} \mathrm{~g}
$$

which is too low to account for the optical properties of a classical 
grain. The mass of a classical grain of three linear dimensions of order 0.5 micron and densities of about $1 \mathrm{~g} / \mathrm{cm}^{3}$ would be about $10^{-14}$ to $10^{-13} \mathrm{~g}$, depending on the shape. Even whiskers could not weigh less than $10^{-18} \mathrm{~g}$.

Whiskers must be treated separately, as follows: Assume a prism of square cross section $a^{2}$ and length $z$, which grows along the $z$-axis, at one end. The growth-rate equation is then

$$
m_{1}(t)=\mu_{1} \exp \left(k_{1} t\right)
$$

where $\mu_{1}$ is the mass of the initial nucleus. The constants $k_{1}$ and $k$ are related by

$$
\begin{aligned}
& k=4 k^{3} \\
& k_{1}=4 k_{0} / a
\end{aligned}
$$

where $k_{0}$ is the rate of accretion in $\mathrm{g} / \mathrm{cm}^{2}-\mathrm{Myr}$, the density of the grain having been assumed to be $1 \mathrm{~g} / \mathrm{cm}^{3}$. Equations (15) and (16) are easily derived by considering the volume and surface of a spherical grain, $\frac{4}{3} \pi r^{3}$ and $4 \pi r^{2}$, and the volume and surface area of a whisker, $z a^{2}$ and $4 a z+2 a^{2} \cong 4 a z$, respectively. In the case of whiskers, it is supposed that atoms are adsorbed, diffuse, and eventually react, and that the products diffuse until they are trapped by the dislocation at one end.

The total radiation dose at age $t$ for a whisker is

$$
D=\mu_{1} \int_{0}^{t} D^{\prime} \exp \left(k_{1} t\right) \mathrm{d} t=\left(\mu_{1} / k_{1}\right) D^{\prime} \exp \left(k_{1} t\right)
$$

The survival equation for whiskers results from considering, again, equations (3) and (4):

$$
k_{1} D_{0} / D^{\prime}>1
$$

or, introducing equation (17):

$$
4 k_{0} D_{0} / a D^{\prime}>1
$$

Survival is therefore favored by small values of $a$. Since equations (15) to (20) are valid only for small values of $a$ (because the term $2 a^{2}$ was neglected in the grain surface) the value

$$
a=10^{-7} \mathrm{~cm}
$$

is assumed. By using $k_{0}=2.5 \times 10^{-8} \mathrm{~g} / \mathrm{cm}^{2}-\mathrm{Myr}$ and the value of $D_{0}$ 
given by equation (8), one arrives at the maximum dose rate compatible with whisker survival

$$
D_{w}^{\prime}=3 \times 10^{21} \mathrm{eV} / \mathrm{g} \cdot \mathrm{Myr}
$$

which is essentially in agreement with the value derived for tridimensional grains (eq. (11)). The maximum mass of a whisker would again be given by equations (13) and (14); i.e., three orders of magnitude smaller than the mass of a whisker of $a=10^{-7} \mathrm{~cm}$ and $z=5 \times 10^{-5} \mathrm{~cm}$ and density close to unity. However, the chance of survival of thicker whiskers may be higher, although it seems that the chance of survival could hardly counterbalance the significantly lower, actual values of the rate of growth. A similar treatment of platelets leads to comparable results.

The point made in a preceding paragraph about having an electron expelled from the grain, carrying out a large fraction of the energy burst $W_{m}$, may be considered again in the case of a whisker. The probability of such a loss would seem, in this case, to be independent of size, because both surface and mass grow at the same rate for a constant cross section. One may then assume, in order to estimate the importance of such a loss, that interaction with the outer layer (about $10^{-8} \mathrm{~cm}$ wide) would lead to the loss of an energetic electron, while interaction inside that layer would transfer the energy $W_{m}$ to the grain. In the assumption of a whisker of square cross section of edge $a=10^{-7} \mathrm{~cm}$, the ratio of the volume of the layer to the total volume of the grain is about 0.4 . In order to account for the loss of energy by the expelled energetic electrons, one may multiply $D^{\prime}$ by the probability of bulk transfer 0.6 and use this value in the survival equation. Although introduction of this factor slightly favors survival, the results remain practically unchanged since the rate of growth is likely to be much smaller.

One may conclude that, in view of the present estimates of the intensity of the galactic radiation-assumed to be uniform throughout space - and the abundance of elements, classical grains of the proper size are unlikely to exist (perhaps with the exception of the very high density $H I$ regions), unless one accepts the idea of reference 11 that a solid grain can result from evaporation of a larger grain.

In the case of a Platt particle, the value of $D_{0}$ should be at least 10 times larger since it would be necessary, in order to destroy the grain, to break chemical bonds. By adopting the minimum value of the galactic specific dose rate of equation (12), one may estimate the minimum value of the growth-rate constant of equation (1) above which a tridimensional Platt particle could survive. The result is

$$
k_{P}=7 \times 10^{-30} \mathrm{~g} / \mathrm{Myr}^{3}
$$

$271-9920-67-17$ 
or $10^{7}$ times smaller than the maximum value provided by the simple theory of growth. This leads to a value of $k_{0}$ that is $10^{7 / 3} \mathrm{smaller}$ than its maximum value. The age of a Platt particle, estimated with this value of $k_{P}$ by neglecting effects that could tend to decrease its size by partial decomposition, would then be of the order of 1 gigayear. Should growth of a Platt particle be interpreted as an increase of the molecular weight by chemical attachment of gaseous matter, it would be reasonable to expect an average activation energy. In the absence of steric factors (entropy of activation close to zero), an average activation energy $E^{*}=1.28 \mathrm{kcal} / \mathrm{mole}$ (about $0.05 \mathrm{eV}$ per impinging atom, equivalent to a kinetic temperature of $640^{\circ} \mathrm{K}$ ) could account for the factor $10^{-7 / 3}$ in $k_{0}$, since the term $\exp \left(-E^{*} / r T\right)$ would have that value at $T=100^{\circ} \mathrm{K}$, the temperature of interstellar gas in $\mathrm{H} I$ regions. One may then conclude that if there is an average activation energy for growth of a Platt particle, it must be less than $1.3 \mathrm{kcal} / \mathrm{mole}$.

\section{FINAL REMARKS}

The least compromising conclusion is, perhaps, that the assumption of a single class of grains may be oversimplifying, if not naïve. There are, however, certain speculative conclusions that may be advanced, subject to a revision of the present knowledge:

1. Classical grains are likely to be in a high degree of structural order, and grow by dislocation-controlled mechanisms leading to platelets and whiskers.

2. Classical grains are unlikely to have trapped radicals, although they may have a steady surface concentration of hydrogen atoms continuously recombining to produce hydrogen molecules that would evaporate. This latter process could provide enough energy for partial evaporation, enriching the grain in heavier elements. Whether or not this mechanism could account for the present size as estimated from optical properties seems difficult to decide.

3. If interaction with galactic radiation does not destroy classical grains at an early age, it could induce polymerization and cross-linking, as well as partial vaporization. Whether or not such a process could lead to Platt particles is still a matter of speculation.

4. Platt particles seem to be more probable, however, if the arguments on interaction with galactic radiation presented in this paper are essen. tially correct. One is then tempted to imagine a Platt particle as an unsaturated molecule, perhaps as old as the universe itself, in dynamic equilibrium with radiation - that would tend to destroy it - and the interstellar gas - that would tend to restore it.

5. There are at least two other possibilities that should be studied; namely: (1) further classical growth around a Platt particle; and (2) 
growth around galactic debris expulsed from $\mathrm{H}$ II regions. This latter possibility would permit the investigator to avoid the puzzling mechanism that gave birth to the nuclei.

\section{REFERENCES}

1. Donn, B.: The Physics and Chemistry of Interstellar Grains. Am. Astr. Soc. Meeting (Lexington, Kentucky), Mar. 1965.

2. LeBedinskY, A. I.: Akademiia Nauk SSSR, Doklady, vol. 92, 1953, p. 507.

3. DonN, B.: Survey of Theoretical and Experimental Research on Interstellar Grains. Lowell Obs. Bull., vol. 4, 1960, p. 273.

4. McMillan, J. A.; and Los, S. C.: Nature, vol. 206, 1965, p. 806.

5. McMillan, J. A.; and Los, S. C.: Hydrazine-Water System II. Nonequilibrium Phase Transformations. J. Chem. Phys., vol. 42, 1965, p. 829.

6. Kimura, H.: J. Astron. Soc. Japan, vol. 14, 1963, p. 374.

7. OORT, J. H.; and van DE HuLST, H. C.: Gas and Smoke in Interstellar Space. Bull. Astron. Inst. Netherlands, vol. 10, 1946, p. 187.

8. SIEgel, S.: Model for $\mathrm{OH}$ Radical Stabilization in Ice at $77^{\circ} \mathrm{K}$ under $\gamma$ Irradiation. J. Chem. Phys., vol. 39, 1963, p. 390.

9. SEITz, F.: The Effects of Irradiation on Metals. Rev. Mod. Phys., vol. 34, 1962, p. 656.

10. Evans, D. E.; Pitrs, D. E.; and Kraus, G. L.: Venus and Mars Nominal Natural Environment for Advanced Manned Planetary Mission Programs. NASA SP-3016, 1965.

11. Cernuschi, F.: The Physics of Cosmic Grains. Astrophys. J., vol. 105, 1947, p. 241. 